\title{
The Longitudinal Distribution of Solar Energetic Particles
}

\author{
T. T. von Rosenvinge ${ }^{1,}$, I. G. Richardson ${ }^{1,2}$, H.V. Cane ${ }^{3}$, E. R. Christian ${ }^{1}$, C. M. S. Cohen ${ }^{4}$, \\ A. C. Cummings ${ }^{4}$, A. W. Labrador ${ }^{4}$, R. A. Leske ${ }^{4}$, R. A. Mewaldt ${ }^{4}$, E. C. Stone ${ }^{4}$, \\ M. E. Wiedenbeck ${ }^{5}$ \\ ${ }^{1}$ NASA/Goddard Space Flight Center, Greenbelt, MD 20771 \\ ${ }^{2}$ CRESST and Dept. of Astronomy, University of Maryland, College Park 20742 \\ ${ }^{3}$ Bruny Island Radio Spectrometer, Bruny Island,Tasmania, Australia \\ ${ }^{4}$ California Institute of Technology, Pasadena, CA 91125 USA \\ ${ }^{5}$ Jet Propulsion Laboratory, California Institute of Technology, Pasadena, CA 91109 USA \\ E-mail: tycho.t.vonrosenvinge@nasa.gov, ian.g.richardson@nasa.gov, \\ hilary.cane@utas.edu.au, eric.r.christian@nasa.gov, \\ cohen@srl.caltech.edu,ace@srl.caltech.edu,ral@srl.caltech.edu, \\ rmewaldt@srl.caltech.edu,labrador@srl.caltech.edu, ecs@srl.caltech.edu, \\ mark.e.wiedenbeck@jpl.nasa.gov
}

Using observations from the High Energy Telescopes (HETs) on STEREO A and B and similar observations from SoHO, near-Earth, we have identified 250 individual solar energetic particle events that include $>14 \mathrm{MeV}$ protons since the beginning of the STEREO mission [1]. Between the end of December 2009, when the STEREO A and B spacecraft were, respectively, ahead and behind Earth by $\sim 65^{\circ}$ in ecliptic longitude, and the end of December 2013, 43 different events were clearly detected at all three locations. The observed intensities of such an event are usually assumed to be Gaussian distributed as a function of the longitudes of the Parker Spiral footpoints at the Sun for each observer. This neglects the fact that the interplanetary magnetic field may have large deviations from Parker Spirals, e.g. due to coronal mass ejections from prior events. Nonetheless, we have fit Gaussians to the peak intensities observed simultaneously at three spacecraft for all 43 events. The Gaussian peak intensity is poorly correlated with the corresponding CME speed and the FWHM is uncorrelated with the CME speed. Surprisingly, however, there appear to be distinctly non-random variations of the FWHM values from event to event.

\section{*Speaker}




\section{Introduction}

One of the primary goals of NASA's STEREO mission is to study the propagation of solar energetic particles (SEPs) from the Sun to $1 \mathrm{AU}$ with a view to better predict SEP intensities at Earth. The STEREO mission utilizes two spacecraft, STEREO-AHEAD and STEREO-BEHIND (aka STEREO-A and STEREO-B), which were launched on a single rocket and then inserted into near-circular orbits around the Sun with mean distances from the Sun of 0.96 AU and 1.04 AU, respectively. The two orbital periods are such that, from shortly after the time of launch in late 2006, STEREO-A (STEREO-B) has been drifting ahead (behind) the Earth by about 22.5 degrees per year. The combined observations of STEREO and near-Earth spacecraft such as SoHO, Wind, and ACE have provided the peak intensities of energetic particle events and coronagraph images of the associated Coronal Mass Ejections (CMEs) from multiple vantage points. CME structure and speeds are best obtained when the CME originates from the limb of the Sun and is projected against the plane of the sky. With three different vantage points it frequently occurs that one of these three views has the CME well-projected against the sky, so the STEREO mission is ideal for relating SEP event properties to CMEs.

Using observations from the High Energy Telescopes (HETs) on STEREO A and B and similar observations from SoHO, near-Earth, we have identified $\sim 250$ individual solar energetic particle events that include $>14 \mathrm{MeV}$ protons since the beginning of the STEREO mission [1]. Between the end of December 2009, when the STEREO A and B spacecraft were, respectively, ahead and behind Earth by $65^{\circ}$ in ecliptic longitude, and the end of December 2013, 43 different events were clearly detected at all three locations. Richardson, et al. [1] analyzed these events with respect to the times of arrival and the peak intensities at each spacecraft of both protons and electrons. In this paper we will expand on the analysis of the longitudinal distributions of protons for the 433 -spacecraft events.

The STEREO mission represents the third major era in which observations have been made at multiple spacecraft to understand the spatial propagation of SEPs. The first era was during the early Pioneer missions [2], and the second was during the Helios-1, Helios-2, and IMP-8 missions. Helios-1 and 2 differed from the STEREO spacecraft in that they covered a much larger range of radial distances from the Sun, R, from $0.33 \mathrm{AU}$ to $1 \mathrm{AU}$.

Lario, et al. [3] analyzed Helios-1 and 2 and IMP-8 data for protons between $4-13 \mathrm{MeV}$ and for $27-37 \mathrm{MeV}$, assuming that all SEP event distributions in $\mathrm{R}$ and longitude phi can be represented by a functional form

$$
\mathrm{j}=\mathrm{j}_{0} \mathrm{R}^{-\mathrm{n}} \exp \left(-\mathrm{k}\left(\mathrm{phi}-\mathrm{phi}_{0}\right)^{2}\right)
$$

Here $\mathrm{j}$ represents the peak particle intensity observed at radial distance R from the Sun and longitude phi, $\mathrm{R}^{-\mathrm{n}}$ represents a power-law decrease of particle intensity with $\mathrm{R}$, $\mathrm{phi}_{0}$ represents the offset in longitude between the parent flare and the centroid of the Gaussian, and $\mathrm{k}$ characterizes the Gaussian dependence of particle intensities on longitude. More specifically, phi is the longitudinal difference between the footpoint at the Sun of the Parker Spiral passing through the observer's longitude and the longitude of the corresponding solar event, e.g. flare location. The Gaussian standard deviation sigma is given by $(2 \mathrm{k})^{-1 / 2}$. We will often refer to the Full Width at Half Maximum (FWHM) of the distribution, which for a Gaussian is 2.355 sigma. Equation (1) contains 4 parameters $\left(\mathrm{j}_{0}, \mathrm{n}, \mathrm{k}\right.$, and $\mathrm{phi} \mathrm{i}_{0}$ ), so measurements of intensity at 3 different spacecraft aren't sufficient to determine all 4 parameters for a given event. As a result, Lario, et al. [3] assumed that all events are characterized by the same values $\mathrm{n}, \mathrm{k}$, and $\mathrm{ph}_{0}$ and performed a statistical analysis of an ensemble of paired intensities to find them. See Lario, et al. [3] for details.

We focus here on observations of $\sim 25 \mathrm{MeV}$ protons made by the High Energy Telescopes [4] on STEREO-A and STEREO-B and similar observations at Earth made by SoHO/EPHIN [5] and SoHO/ERNE [6]. STEREO-A has a mean distance from the Sun of $0.962 \mathrm{AU}$ (perihelion $=0.956 \mathrm{AU}$, aphelion $=0.967 \mathrm{AU}$ ), whereas STEREO-B has a mean distance from the Sun of $1.043 \mathrm{AU}$ (perihelion = 
1.00 AU, aphelion = 1.086 AU. If we use the radial dependences reported by Lario, et al. [3], the differences between intensities at $1 \mathrm{AU}$ and at the actual radial distances of the STEREO spacecraft can be expected to be $<\sim 10 \%$. Since this variation is small compared to the azimuthal variations, we have treated STEREO-A and STEREO-B as both being at 1 AU (i.e. we take $n=0$ in equation (1))

\section{Fitting Method}

For each energetic particle event we wish to find values of $\mathrm{j}_{0}$, $\mathrm{k}$ and $\mathrm{phi}_{0}$ which correspond to an underlying Gaussian passing exactly through the points (phi, j) for each of the three spacecraft. This approach ignores the active-region latitude, assumes that particles only propagate in longitude, and assumes that the intensity drops off with longitude corresponding to a Gaussian profile with peak at phi ${ }_{0}$, all deficiencies shared with the Lario, et al. Method [3]. There is no a priori reason or verification that a Gaussian is the right underlying function, and indeed other authors have used other functions, notably an exponential fall off of intensity with increasing longitudinal separation between the flare and the Parker Spiral footpoint [7], [8]. Another complication is that a Gaussian is defined for a one-dimensional variable extending from - infinity to + infinity, whereas longitude ranges from, let's say, -180 to + 180 degrees. For example, longitude values at -170 degrees and +170 degrees are arithmetically separated by 340 degrees, whereas spatially these two longitudes are only separated by 20 degrees. If we can find a procedure which can mathematically handle this, we will be able to find a value for each parameter for each solar event. We can then try to correlate the Gaussian parameters with CME parameters for each event or look at their time histories, neither of which can be done with the Lario, et al. method [3]. We can also compare the averages of these parameters with the results of [3]. Note that our approach assumes that the underlying distribution is Gaussian but, unlike the Lario, et al. method [3], it does not assume that this Gaussian has a fixed FWHM and phi $_{0}$ for all events.

We have found two separate algorithms to find the underlying Gaussian distribution. The first uses the standard Gaussian fitting routine with x extending from - infinity to + infinity and 3 different cases: the first case is with the 3 spacecraft foot-points located at longitudes in the range -180 to +180 degrees; the $2^{\text {nd }}$ case is the same as case 1 except that the eastern-most foot-point longitude is increased by 360 degrees; and the $3^{\text {rd }}$ case is the same as case 1 but the western-most foot-point longitude is decreased by 360 degrees. Cases where the middle intensity is lower than both of the other two will not have solutions. From the remaining cases, select the one with the smaller FWHM. The second method is a Monte Carlo search where random parameter values in the Gaussian parameter space are used to compute corresponding intensities at the three spacecraft locations; the goodness of fit can be measured as the sum of the squares of the percentage differences between the three Gaussian intensities and the three observed intensities. Then search for the parameter set which gives the best fit. This is broken into 3 phases: first use random parameters selected from the whole parameter space and save the ones for, let's say, the 50 best fits; then reduce the search space to match the ranges of parameters in the best 50 fits and search the reduced space as before; then repeat the previous step. Both algorithms give the same results. The advantage of the Monte Carlo method is that it is readily extended to consider that particles can propagate from the flare to the observer by going both east and west. The method described so far only considers particles passing either east or west, depending on which is the shorter path. These two approaches only differ when the two paths are very nearly the same in longitudinal extent.

\section{Observations}

Peak intensities and spacecraft longitudes for each 3-spacecraft event are taken from Table 1 in Richardson et al. [1] The Parker Spiral foot-points are obtained using the observed solar wind speed at each spacecraft location at the start of the event (Galvin, et al. [9] for STEREO; Lin et al., [10] from Wind near Earth).

The associated solar events were identified using: movies from SoHO, SDO, and STEREO; CME observations and movies from SoHO and STEREO; H- $\alpha$ flares; GOES X-ray flares; and radio emissions from WIND/WAVES and STEREO/SWAVES. In every case, an unambiguous association has been made. 
We now present results from the functional fitting of the data as described in Section 2.

Figure 1 shows the Gaussian peak intensity versus CME speed. Here we use the CME speed reported by the Catholic University of America based on observations from SoHO

(http://cdaw.gsfc.nasa.gov/CME_list). Each data point in the plot is numbered by the number of the event, 1 - 43. Note that the intensity can differ by four orders of magnitude for a fixed CME speed. This weak correlation is well known. Assuming that particles are accelerated by the shock, one might expect a better correlation. Lario and Karelitz [11] have made plots of the intensity observed at Earth for well-connected proton events in 3 different energy ranges (9-15 MeV, 15-40 MeV, and 40-80 MeV). Their plots (Figure 5) are similar to Figure 1 but have a more well-defined upper limit of intensity versus CME speed for each energy band. This may be in part due to the fact that we are restricted to 3-spacecraft events, which tends to exclude small events. Figure 2 shows that the peak intensity and FWHM for the 43 events are essentially uncorrelated. This is perhaps not too surprising considering that the calculation of the FWHM depends on the peak intensity.

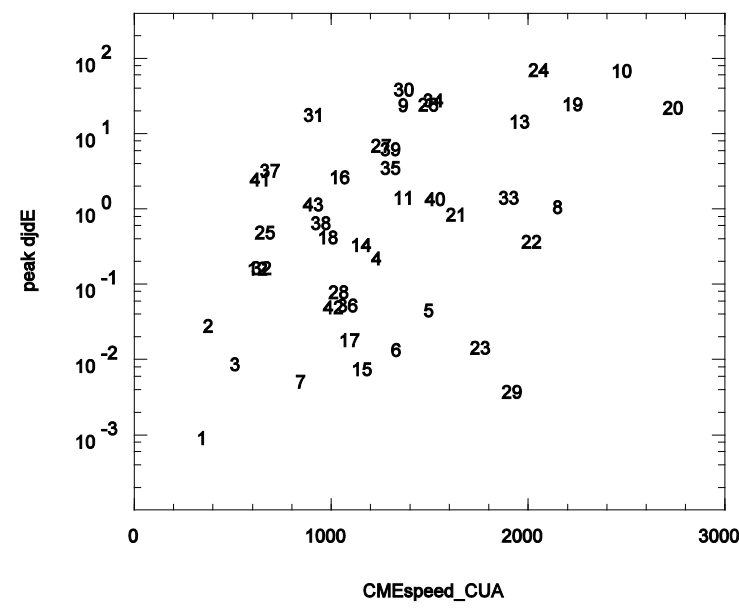

Figure 1. Shows the peak of the fitted Gaussian versus CME speed in $\mathrm{km} / \mathrm{sec}$ for each of 43 different 3spacecraft events.

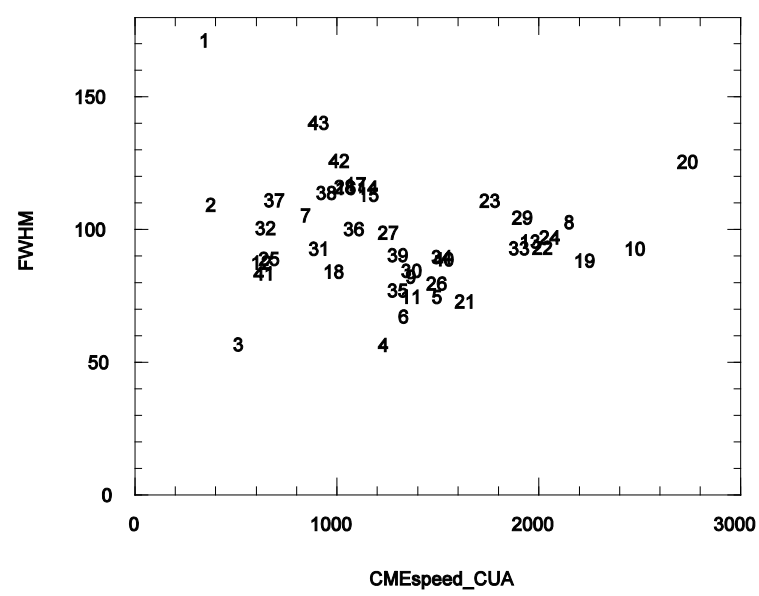

Figure 3. Shows the Gaussian-fit FWHM plotted versus the CME speed for each event.

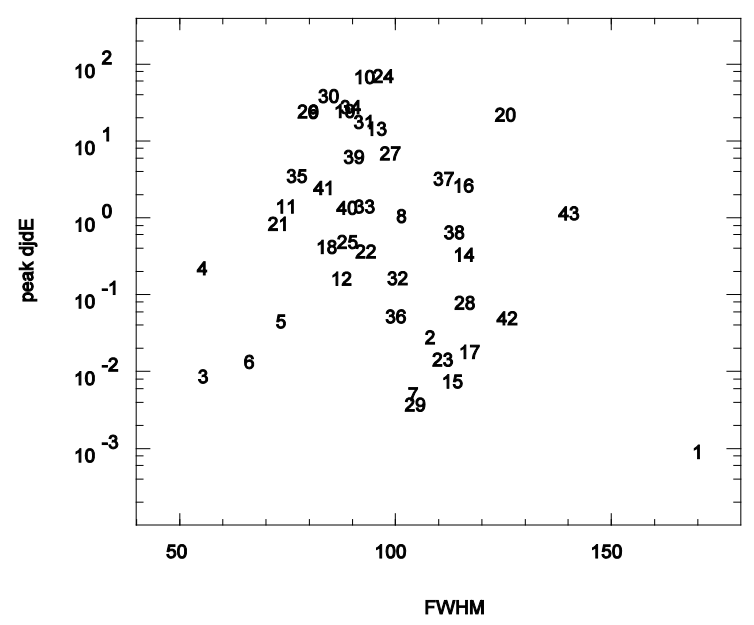

Figure 2. Shows the Gaussian-fit peak intensity plotted versus the FWHM of the fit for each event.

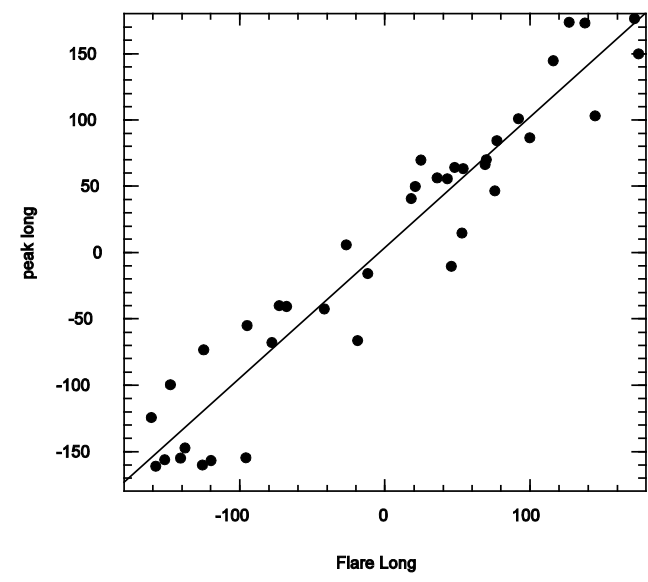

$y=a^{4} x+b ; w$ td $=0.883+0.048$

Figure 4. Shows the Gaussian peak longitude versus the flare/active region longitude. The parameters for the straight line fit to the data are on the upper right hand side. 
Figure 3 shows that the FWHM is independent of the CME speed. At first glance this may appear to be counter-intuitive. For a given detection sensitivity, more intense events will be visible over a wider range of longitudes than less intense events. However this doesn't mean that the FWHM has to be any different.

Figure 4 shows that the Gaussian peaks are, on average, consistent with being at the same longitude as the flare/active region.

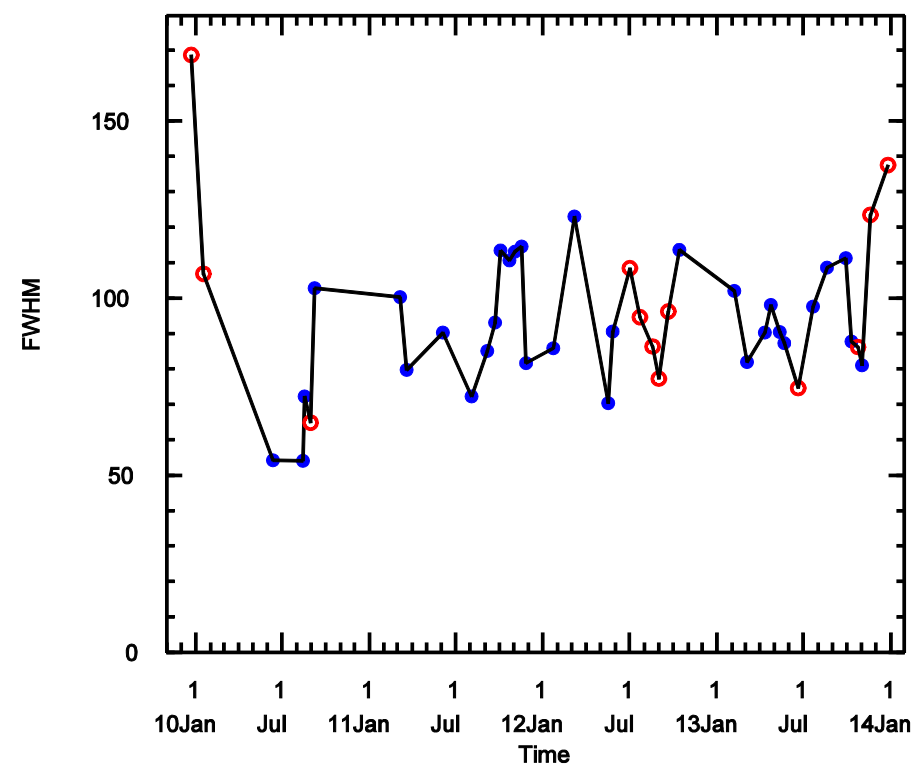

Figure 5 (left). The FWHM for each event plotted versus the time of the event. The blue (solid) circles indicate active regions in the Sun's northern hemisphere and red (open) circles are for the southern hemisphere.

Figure 5 shows that most of these events occurred in the northern hemisphere of the sun. The five consecutive red points correspond to a single long-lived active region in the southern hemisphere. Figure 5 also shows an unexpected result. After March 2011, the time history shows a succession of approximately periodic peaks.
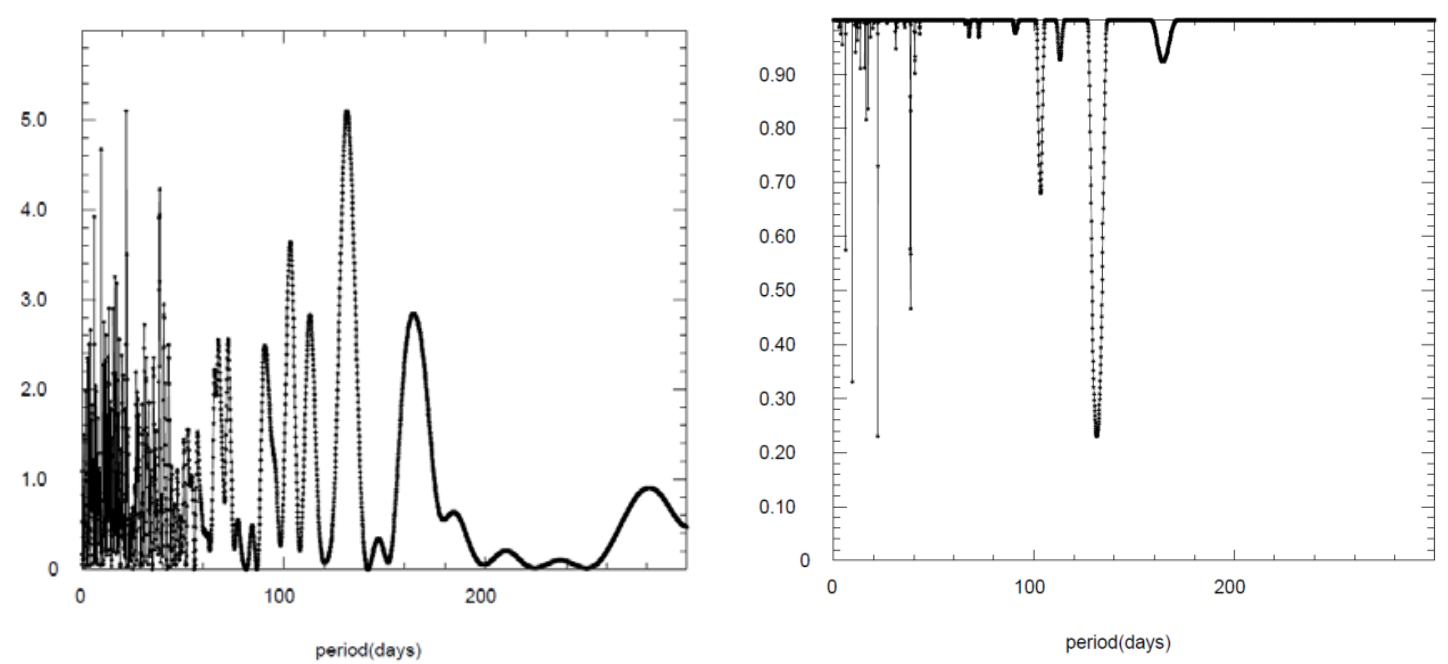

Figure 6. (left) Power versus period in days, (right) the probability that power in the left hand plot at a given period has occurred at random.

In Figure 6 we show results of a Lomb power spectrum analysis [12]. This type of analysis is particularly suited to irregularly spaced data. The left side of Figure 6 shows that there is a peak in the power spectrum at approximately 132 days ( 4.2 months). The right side of Figure 6 shows the probability of the power in the left hand plot being random. We see that there is a $20 \%$ chance of the peak at 132 days being random. A reasonable view is that the peaks are real but may signal a cause which is not fully periodic. Periodicities in solar gamma-ray events (Rieger, et al. [13]), solar electron events 
(Evenson et al. [14]), and in the interplanetary magnetic field (Cane et al. [15]) have previously been reported to have periods of $\sim 153$ days, however significantly different periods have been reported at other times. Whether these periodicities are related to the approximate periodicity reported here is unknown.

\section{References}

[1] I. G. Richardson, T. T. von Rosenvinge, H. V. Cane et al., >25 MeV Proton Events Observed by the High Energy Telescopes on the STEREO A and B Spacecraft and/or at the Earth During the First Seven Years of the STEREO Mission, Solar Physics, 289, doi: 10.1007/s11207014-0524-8 (2014).

[2] K. G. McCracken and U. R. Rao, Solar Cosmic Ray Phenomena, Space Science Reviews 11,155, (1970).

[3] D. Lario, M.-B. Kallenrode, and R. B. Decker et al., Radial and Longitudinal Dependence of Solar 4-13 and 27-37 MeV Proton Peak Intensities and Fluences: Helios and IMP 8 Observations, Astrophysical Journal, 653, 1531 (2006).

[4] T. T. von Rosenvinge, D. V. Reames, R. Baker, et al., The High-Energy Telescope for STEREO, Space Science Reviews, 136, doi: 10.1007/s11214-007-9300-5 (2008).

[5] R. Müller-Mellin, H. Kunow, V. Fleissner, et al., Comprehensive Suprathermal and Energetic Particle Particle Analyzer, Solar Physics, 162, 483 (1995).

[6] J. Torsti, E. Valtonen, M. Lumme, et al., Energetic Particle Experiment ERNE, Solar Physics, 162, 505 (1995).

[7] S. W. Kahler, Journal of Geophysical Research, 87, 3439 (1982).

[8] R. E. McGuire, M. A. I. Van Hollebeke, and N. Lal, Proc. $18^{\text {th }}$ International Cosmic Ray Conference, 10, 353 (1983).

[9] A.B. Galvin, L.M. Kistler, M.A. Popecki, et al., The Plasma and Suprathermal Ion Composition (PLASTIC) Investigation on the STEREO Observatories, Space Science Reviews, 437, doi: 10.1007/s11214-007-9296-5 (2008).

[10] R.P. Lin, K.A. Anderson, S. Ashford, et al., A Three-Dimensional Plasma and Energetic Particle Investigation for the WIND Spacecraft, Space Science Reviews, 71, doi: 10.1007/BF00751328 (1995).

[11] D. Lario and A. Karelitz, Influence of interplanetary coronal mass ejections on the peak intensity of solar energetic particle events, Journal of Geophysical Research 119, 4185 - 4209, doi: 10.1002/2014JA19771 (2014).

[12] N. R. Lomb, Least-squares frequency analysis of unequally spaced data sets, Astrophys. Sp. Sci., 39, 447 (1976).

[13] E. Rieger, G. H. Share, D. J. Forrest, G. Kanbach, C. Reppin, and E. L. Chupp, A 154 day periodicity in the occurrence of hard solar flares?, Nature 312, 623 (1984).

[14] P. Evenson, P. Meyer, S. Yanagita and D. J. Forrest, Electron-Rich Particle Events and the Production of Gamma-Rays by Solar Flares, ApJ 283, 439 (1984).

[15] H. V. Cane, I. G. Richardson, and T. T. von Rosenvinge, Interplanetary magnetic field periodicity of 153 days, Geophys. Res. Letters, 25, 4437, (1998). 